\title{
The Protection of Frequencies for Radio Astronomy
}

\author{
R. L. Smith-Rose \\ President, International Scientific Radio Union
}

(Received November 5, 1962)

\begin{abstract}
The International Telecommunications Union in its Geneva, 1959 Radio Regulations recognises the Radio Astronomy Service in the two following definitions:

No. 74 Radio Astronomy: Astronomy based on the reception of waves of cosmic origin.

No. 75 Radio Astronomy Service: A service involving the use of radio astronomy.

This service differs, however, from other radio services in two important respects.

1. It does not itself originate any radio waves, and therefore causes no interference to any other service.

2. A large proportion of its activity is conducted by the use of reception techniques which are several orders of magnitude more sensitive than those used in other radio services.

In order to pursue his scientific research successfully, the radio astronomer seeks protection from interference first, in a number of bands of frequencies distributed throughout the spectrum; and secondly, more complete and specific protection for the exact frequency bands in which natural radiation from, or absorption in, cosmic gases is known or expected to occur.

The International Regulations referred to above give an exclusive allocation to one frequency band only-the emission line of hydrogen at 1400 to $1427 \mathrm{Mc} / \mathrm{s}$. In all other cases, allocations are made on a basis of sharing with other radio services.

Considering the expensive nature of the equipment in use and under development by radio astronomers throughout the world, it is important that the protection of certain bands of frequencies for this science should receive serious attention by all those engaged in both local and worldwide radio services.
\end{abstract}

\section{Introduction}

The science of astronomy using optical telescopes to explore the heavens is some centuries old: the classical works of Copernicus, Galileo, Kepler, and Newton form milestones in its development in the 15 th to 18th centuries. At first the scientific observational techniques were limited to wavelengths within the visual range, since they were dependent upon the detecting power of the human eye. But the application of electrical recording and measuring instruments allowed an extension to both the shorter and longer wavelengths comprising the ultraviolet and infrared portions of the spectrum: and by and large the photographic camera has now replaced the eye of the human observer.

In order to ensure that the electromagnetic waves radiated from the galaxy, stars, and planets, suffer a minimum of absorption in traveling through the earth's atmosphere, it has become an accepted practice that astronomical observatories are situated in locations where clouds in the atmosphere are a minimum; and, usually the observatory is installed at as high an altitude as possible in the area selected. Moreover with the growth of our civilization, and the corresponding increase of artificial illumination outdoors, the astronomer is faced with the quite serious problem of locating his observatory at a place where the background illumination from terrestrial sources is reduced to a minimum.

Presented at the Joint Meeting of URSI (Canadian and United States National Committees) and IRE held in Ottawa 15-17 October 1962.
It has long been quite unnecessary to justify the pursuit of the science of astronomy, for its many applications have become so obviously necessary in the conduct of modern civilization. The derivation and maintenance of time standards; the navigation services at sea, on land, and more recently in the air, are all dependent in the long run on astronomical observations; and on the knowledge built up in the past by the work of astronomers all over the world. But there still remains the fact that the basic range of wavelengths available for use by the optical astronomer is limited to approximately the band 1000 to 30,000 A, a mere five octaves (corresponding to wavelengths from $10^{-7}$ to $3 \times 10^{-6} \mathrm{~m}$; or frequencies from $3 \times 10^{15}$ down to $10^{14} \mathrm{c} / \mathrm{s}$ ). As will be seen, the development of radio astronomy has added another 12 octaves of the electromagnetic wave spectrum to the performance of the tools and apparatus available for scientific research.

\section{Radio Astronomy}

The science of radio astronomy is now over 20 years old, for it was in 1931 that Karl G. Jansky of the Bell Telephone Laboratories, U.S.A., first detected radio waves received from the Milky Way; later to be known in the radio field as "galactic noise." Some 10 years later, during the second World War, two distinct types of radio emission from the sun were observed: Hey and his colleagues [1946] detected intense and variable radiation on meter wavelengths; while Southworth [1945] observed 
steady emission on centimeter wavelengths. At about the same time Reber [1942, 1944] observed radiation from the sun on a frequency of $160 \mathrm{Mc} / \mathrm{s}$ (corresponding to a wavelength of about $1.9 \mathrm{~m}$ ). A little later, another branch of radio astronomysometimes designated nowadays as "radar astronomy" - started following the experimental demonstration by Hey and Stewart [1946] that radar echoes could be received from meteor trails.

Since these early discoveries, the new science of radio astronomy has developed to an enormous extent in all parts of the world; and a correspondingly extensive literature has developed in books and scientific journals. It has been demonstrated that radio waves from cosmic sources-or from the continuum as it is termed-can be received over a frequency range from a few megacycles per second to more than $30 \mathrm{Gc} / \mathrm{s}$, covering over 12 octaves of the electromagnetic wave spectrum. Apart from observations on such powerful sources as the sun, the radio astronomer is confronted with the recording and study of very weak radiations, the strength of which is several orders of magnitude less than those used in the practice of radio communications. To achieve some measure of success the radio astronomer has, in the first place, had to design and construct large and highly directive antenna systems in order to collect enough energy to detect, and to enable him to locate as accurately as possible the direction of the source of the radiation. Secondly, he has had to use the most advanced techniques in the development of the highly sensitive receiving equipment required for his work.

Following the early work referred to above, two further discoveries have emerged as playing a fundamental part in the pursuit of radio astronomy. After the observation by Hey and his colleagues of fluctuations in the intensity of cosmic radio waves from the constellation of Cygnus, it was shown by Bolton and Stanley [1948] that the fluctuations were associated with an intense radio source which, although in the same region, did not appear to be identifiable with any optical source. It thus became clear that there were discrete sources of cosmic radio waves, from which the visual radiation was negligible, and these have become designated as "radio stars."

The second discovery was that of the atomic hydrogen spectral line in emission from the Galaxy.

\subsection{Hydrogen Line at 1400 to $1427 \mathrm{Mc} / \mathrm{s}$}

For more than half a century, astronomers have been interested in the radiation from interstellar space, and particularly in the emission and absorption properties of the gases which, with a luminous belt of stars, form the Galaxy or Milky Way, the average brightness of which is about twice that of the remaining portion of the sky.

In 1945, van de Hulst suggested the possibility that hydrogen gas in the galactic clouds might be a source of radio frequency waves; and this was endorsed by Shklovsky [1949] who concluded that the radiation would be of sufficiently high intensity to be detected. Two years later, in 1951, Ewen and Purcell of Harvard announced their successful detection of this radiation from the Galaxy; and this was immediately confirmed by the independent observations of Muller and Oort [1951] in Leiden, and of Pawsey [1951], and Christensen and Hindman [1952] in Australia. In the past decade, a great deal of research has been conducted by radio astronomers on the hydrogen band radiation received from various parts of the Universe; and this is undoubtedly making a considerable and worthwhile contribution to our knowledge of the space around our earth. The need for this work to be protected from interference was recognized at the Geneva, 1959, meeting of the International Telecommunication Union, which assigned the band of frequencies 1400 to $1427 \mathrm{Mc} / \mathrm{s}$ (wavelength $21 \mathrm{~cm}$ ) on a primary basis for the exclusive use of the radio astronomy service. This is referred to in a later section of the paper.

\subsection{Deuterium Line at 322 to $329 \mathrm{Mc} / \mathrm{s}$}

Following the research leading to the discovery of the hydrogen line radiation, radio astronomers predicted the existence of radiation from deuterium gas - an isotope of hydrogen of twice its atomic weight - in a band of frequencies 322 to $329 \mathrm{Mc} / \mathrm{s}$. It is to be expected from the much lower concentration or "abundance" of this gas, that the radiation from it will be more difficult to detect and identify; but it is natural that the radio astronomer should expect to be encouraged to do so. Shklowsky in 1952 suggested that radiation from deuterium at a frequency of $327 \mathrm{Mc} / \mathrm{s}$ (wavelength $91.6 \mathrm{~cm}$ ) should be detectable even though it was to be expected that the concentration of interstellar deuterium is only about $1 / 1000$ that of hydrogen. Experiments made in 1954 in Australia by Stanley and Price (described in 1956), appeared to confirm that the concentration was less than this value.

Early in 1957, the position was reviewed by Adgie and Hey, who referred to the measurements made during 1954-55 by Gemantzev, Stankevitch, and Troitsky, indicating a deuterium concentration relative to hydrogen of about one part in 300 , an abundance ratio some 20 times greater than the terrestrial value. But new experiments conducted by Adgie and Hey, with equipment estimated to have greater sensitivity than that used by Stanley and Price, led them to conclude that the abundance of deuterium does not exceed 1/2000 that of hydrogen. Two further attempts were made in 1959 by Adgie using the $250 \mathrm{ft}$ radio telescope at Jodrell Bank, England, to detect the $327 \mathrm{Mc} / \mathrm{s}$ radiofrequency line of galactic deuterium; but even when the bandwidth of the receiver was reduced to $6 \mathrm{kc} / \mathrm{s}$, the experiments gave a negative result. An investigation conducted at the U.S. National Radio Astronomy Observatory at Green Bank, W.Va., led S. Weinreb [1962] to conclude that the ratio of deuterium to hydrogen is about $1 / 13000$, or about half the terrestrial value.

Further critical experiments will become possible when new radio telescopes of greater sensitivity have 
been built; and when the deuterium line can then be sought as an absorption band in the continuum of radiation from a distant discrete source. It is of fundamental importance that such experiments should be carried out, and radio astronomers should be encouraged to do so by giving them the utmost protection from interference by other users of this band of frequencies.

\subsection{Radar Āstronomy}

In concluding this section, a brief reference may be made to the use of radar techniques in astronomy. Such techniques have been successfully used in various parts of the world for the scientific investigation of aurora and meteor trails. For this purpose the astronomer takes his place with other users of the spectrum in that he needs to use some bands of frequencies in which to emit radiation so that he may study the echoes reflected from the natural phenomena mentioned above. There is, however, no accepted international definition of the term "Radar Astronomy;" and indeed there is some difference of opinion among the astronomers themselves as to the need for such a term. It is likely that proposals will come before the forthcoming Plenary assembly of CCIR in 1963 which may clarify this aspect of the subject.

\section{International Organization of Radio}

As we have seen above, the science of radio astronomy has developed rapidly, particularly over the past decade or so. But over the same period, other users of the radiofrequency spectrum have also been active in demanding increased facilities for all types of communications, private and public, civil and military, sound and television broadcasting, and for such associated applications as radar and navigational aids for ships and aircraft. In order to avoid a state of confusion, and possibly chaos, which would otherwise arise in the radio field, international organizations confer at intervals on both the technical and administrative problems involved.

\subsection{International Telecommunication Union}

It became clear in the early years of the present century, that the then new service of radio communications would need to be protected at both national and international levels, and rules drawn up to govern its use. Following earlier international meetings, it was the Atlantic City Conference of 1947 that formed the present constitution and practice of the International Telecommunication Union (ITU), which had then become one of the specialized agencies of the United Nations, and had attained a membership of 72 countries. At this conference a frequency allocation table was drawn up for the three regions into which the world was divided for radio communication purposes.

The next full administrative Radio Conference was held in Geneva in 1959; and in the intervening 12 years, major developments had taken place in the use of radio for communication by ionospheric and tropospheric scatter, for aerial and marine navigation, and for scientific purposes including radio astronomy and communication with, and control of, artificial earth satellites and space vehicles. In addition, the demand for radiofrequency channels for fixed and mobile services and for all forms of longdistance communications had steadily increased. Although with improved techniques, many users were able to reduce the width of the frequency channel required to carry the information, this reduction in spectrum space was more than offset by the great increase in demand for further channels.

The Geneva conference [1959] was attended by upwards of 700 people, including delegates from some 90 member countries of ITU, and representatives of international organizations including the International Scientific Radio Union (URSI), the International Astronomical Union (IAU) and the International Committee on Space Research (COSPAR). The new frequency allocation table which resulted from this conference covered the range $10 \mathrm{kc} / \mathrm{s}$ to $40,000 \mathrm{Mc} / \mathrm{s}(40 \mathrm{Gc} / \mathrm{s})$, nearly four times that of the 1947 Atlantic City table.

Before referring to the frequencies which are of direct interest to radio astronomers it will be convenient here to refer to some other organizations associated with ITU.

\subsection{International Radio Consultative Committee}

The International Radio Consultative Committee (CCIR) was established in 1927 for the purpose of studying technical questions which are of interest in the development and practice of radio communications and which are submitted by the national administrations and private operating agencies constituting its membership. It deals by separate study groups with the technical problems of sending and receiving equipment, with the propagation of radio waves of all frequencies and under all conditions, with interference from natural and manmade disturbances and with the specification of conditions and tolerances appropriate to all applications of radio. The function of CCIR is limited to the expression of an opinion on the various technical questions studied and the transmission of conclusions and recommendations to ITU. In brief, it may be said that the object of its work is to see that the radiofrequency spectrum is divided among the users in the most economical and efficient manner possible, to facilitate the conduct of the individual services required with the minimum of interference.

\subsection{Inter-Union Committee on Frequency Alloca- tions for Radio Astronomy and Space Science}

Although at meetings of CCIR in the past, URSI, IAU, and more recently, COSPAR have been able to attend in an observing capacity, it was at the General Assembly of URSI in London in 1960, that a more definite step was taken to coordinate 
the future requirements of frequency channels for both radio astronomy and space science. At this meeting, an Inter-Union Committee on Frequency Allocations for Radio Astronomy and Space Science known more colloquially as IUCAF, was formed with equal membership from the constituent bodies, URSI, IAU, and COSPAR. Dr. J. F. Denisse, the French astronomer at the Meudon Observatory, was appointed Chairman, and the present writer has undertaken the services of Secretarv-General [Smith-Rose, 1961]. The constitution and terms of reference of the committee include the coordination of the requirements of the three constituent bodies, the formulation of proposals for frequency allocations to meet these requirements, and cooperation with CCIR with the view of ensuring that these proposals are placed on the agenda of ITU.

This Inter-Union Committee has already met in Brussels, London, and Amsterdam, and it will convene again in Geneva in November. The Chairman and Secretary-General attended the interim meeting of CCIR Study Group IV in Washington in March 1962, and active steps are being taken to ensure that the subject of frequency allocations for radio astronomy is included in the agenda of the Extraordinary Administrative Radio Conference scheduled for the autumn (fall) of 1963 .

\section{Frequencies for the Radio Astronomy Service}

At the International Administrative Radio Conference, 1959 , two definitions relevant to radio astronomy were drawn up and printed in chapter 1, "Terminology," of the Radio Regulations [1959]. These are:

74. Radio astronomy: Astronomy based on the reception of radio waves of cosmic origin.

75. Radio astronomy service: A service involving the use of radio astronomy.

From these definitions, it is clear that radio astronomy differs from all other radio services in that it does not make use of radio emissions from manmade sending stations. Its work is based on the reception and study of the natural emissions of sources in outer space. Thus the radio astronomer can cause no interference to other services; but he justifiably seeks the exclusive use of certain bands of frequencies in the spectrum, so that he may pursue his studies free from interference from the transmitting stations of other services. Furthermore, since the radiation he is seeking to measure is, in many cases extremely weak, he must necessarily use advanced techniques - large aerial systems and very sensitive receivers. But the expense and effort required to install and operate such systems can be justified only if he is assured of adequate protection from interfering emissions.

Details of all the frequencies allocated to radio astronomy in the Radio Regulations, Geneva, 1959, are given in the table forming appendix A. They fall into two distinct classes.

\subsection{Line Frequencies or Bands Arising from Natural Phenomena}

In the first place, the radio astronomer seeks full protection in those bands where naturally occurring radiation exists, and which therefore cannot be changed by international discussion. As already mentioned in section 2.1, the 1959 Radio Regulations give an exclusive allocation of the band 1400 to 1427 $\mathrm{Mc} / \mathrm{s}$ (the hydrogen line) to radio astronomy; although in certain European countries and the U.S.S.R. this band is also allocated to the fixed service and the mobile, except aeronautical mobile, service. The next band in which there are strong scientific reasons to expect the occurrence of natural radiation is the deuterium line at 322 to $329 \mathrm{Mc} / \mathrm{s}$. In this case the frequencies are shared with fixed, mobile, and aeronautical radionavioation services in all parts of the world. Efforts are being made in the European region to seek the cooperation of administrations using these other services so that radio astronomers may conduct observations in this band free from harmful interference.

Apart from these two bands (hydrogen and deuterium) a number of other bands in which naturally occurring radiation is to be expected have aroused the interest of radio scientists. These range from the $\mathrm{OH}$ line at 1645 to $1675 \mathrm{Mc} / \mathrm{s}(1.645-$ $1.675 \mathrm{Gc} / \mathrm{s})$, to those associated with oxygen, ozone, and other gases at certain frequencies between about 30 and $100 \mathrm{Gc} / \mathrm{s}$. It is also desirable that there should be opportunities to investigate the "windows" in the earth's atmosphere where the absorption of extremely high frequency radio waves passes through a minimum. Such windows are expected to be found at about 30 and $90 \mathrm{Gc} / \mathrm{s}$, and at higher frequencies.

\subsection{Frequencies in the Continuum Background}

For the second and more general class of research, radio astronomers have stressed their need to use a minimum of about 10 or 12 bands of frequencies distributed throughout the spectrum. The approximate positions of some of these have been indicated as follows: $40,80,160,640,2560,5120$, and 10,240 $\mathrm{Mc} / \mathrm{s}$. While the claim to bands near most of these is recognized by footnote references in the 1959 Radio Regulations, in all cases the frequencies are allocated on a basis of sharing with the operators of other radio services. This is very unsatisfactory to the radio astronomer, who is naturally very anxious to press his case for a more substantial measure of protection.

A very helpful move in the right direction was made at the European Broadcasting Conference at Stockholm in 1961, where it was agreed that so far as the European region was concerned, no major television broadcasting stations should be planned to operate in the band 606 to $614 \mathrm{Mc} / \mathrm{s}$, so that this might be kept freely available for the radio astronomy service. This agreement was a logical consequence of recommendation No. 32 (par. 3) of the Radio Regulations. Paragraph 2 of the same recommenda- 
tion refers to the possibility of making a firm allocation in the range 37 to $41 \mathrm{Mc} / \mathrm{s}$, where frequencies at 38 and $41 \mathrm{Mc} / \mathrm{s}$ are being used, or proposed for use, by radio astronomers.

\subsection{Degree of Protection Required}

It has already been stated that the radio astronomer carries out his research by receiving radio emission from natural sources. Apart from certain high power radiators such as the sun, the observation of these emissions requires the use of receiving techniques of the utmost sensitivity. It is natural to find that the radio astronomer, like his colleague who uses optical instruments, endeavors to locate his observatory where radio interference from manmade sources is expected to be a minimum. For example, the site for the U.S. National Radio Astronomy Observatory was carefully chosen to be as free as possible from radio interference. Findlay [1958] has described the results of measurements and the precauticns taken to secure protection for the future. Similarly Blum, Denisse, and Steinberg [1958] have described the site of the NANCY radio astronomy field station of the Meudon Observatory in France as having been located 120 miles from Paris to secure the utmost reduction in manmade interference.

Radio astronomers are steadily compiling systematic measurements of the flux density received from a variety of sources; and a few figures may be quoted to indicate the order of sensitivity at which their equipment is operating. For example, in a lecture published in 1958, M. Ryle gave two tables of results of radio flux density as received from galactic and extragalactic sources respectively. The former ranged from 1.1 for a Supernova to 220 for Cassiopeia $A$, the unit in each case being $10^{-24}$ watts per square meter per cycle per second. For the latter - the extragalactic sources - the values quoted range from 0.04 to $133 \times 10^{-24} \mathrm{wm}^{-2}(\mathrm{c} / \mathrm{s})^{-1}$. For corresponding measurements at the Harvard Radio Astronomy Station, Texas, Maxwell, Swarup, and Thompson [1958] assess the intensity of the quiet sun in the range 400 to $2000 \times 10^{-24} \mathrm{wm}^{-2}(\mathrm{c} / \mathrm{s})^{-1}$. Wells [1958] has described measurements on Cassiopeia A, indicating a flux density of 200 to $500 \times 10^{-24} \mathrm{wm}^{-2}$ $(\mathrm{c} / \mathrm{s})^{-1}$; while Roman and Yaplee [1958] give a measured range of 1 to $60 \times 10^{-24} \mathrm{wm}^{-2}(\mathrm{c} / \mathrm{s})^{-1}$ for the flux received from various discrete sources; Mayer, McCullough, and Sloanaker [1958] at the U.S. Naval Research Laboratory, have measured the radiation from the planets Venus, Jupiter, and Mars, as in the order of 0.1 to $1 \times 10^{-24} \mathrm{Wm}^{-2}(\mathrm{c} / \mathrm{s})^{-1}$. More recently Mills, Slee, and Hill [1961] have published catalogues of radio sources observed at Sydney, Australia. In the latest of these, the flux density ranges from about 0.07 to $40 \times 10^{-24} \mathrm{wm}^{-2}(\mathrm{c} / \mathrm{s})^{-1}$.

These examples of experimental measurements indicate that the radio astroncmer is now equipped to detect the radiation flux from natural sources down to a minimum of the order of $10^{-25}$ or less watts per square meter in a bandwidth of $1 \mathrm{c} / \mathrm{s}$. Even on the most remote and quiet sites selected for observatories, the strength of intermittent radio signals from manmade stations not infrequently exceed this value by at least 10 or $20 \mathrm{db}$. As a comparative figure, it may be mentioned that the power flux being received in England on the transatlantic radio link by way of Telstar is of the order of $10^{-17} \mathrm{w} \mathrm{m}^{-2}(\mathrm{c} / \mathrm{s})^{-1}$.

What the radio astronomer is seeking is better protection for his observations made at the very low flux density he has to work with. It is suggested that international bodies, such as URSI and CCIR, should work towards the achievement of a tolerable level of interference at observatories of not more than $20 \mathrm{db}$ below $10^{-24} \mathrm{w} \mathrm{m}^{-2}(\mathrm{c} / \mathrm{s})^{-1}$. In this way the radio astronomer will be able to pursue his observations and research to the ultimate benefit of our knowledge of the space around our earth, and the establishment of continuously expanding space communications in the future.

With a view to ensuring that this matter receives adequate attention at international conferences, the Inter-Union Committee referred to in section 3.3 has drawn up the two recommendations given in appendix B. It is hoped that the CCIR will be able to endorse these recommendations so that they may be brought before the next Administrative Radio Conference to be held in the autumn of 1963 .

\section{Appendix A. Review of Frequencies Assigned to Radio Astronomy in Radio Regulations, Geneva, 1959}

\begin{tabular}{|c|c|c|c|}
\hline No. & Frequency band & $\begin{array}{l}\text { Applicable to } \\
\text { regions }\end{array}$ & $\begin{array}{c}\begin{array}{c}\text { Reference in radio } \\
\text { regulations } \\
\text { Rec. }= \\
\text { Recommendation } \\
\mathrm{F}=\text { Footnote }\end{array}\end{array}$ \\
\hline 1 & $\begin{array}{l}M \mathrm{Mc} / \mathrm{s} \\
\text { Standard frequency } \\
\text { guard bands } 2.5,5 \text {, } \\
10,15,20 \text {, and } 25 \text {. }\end{array}$ & 1,2 , and $3 \ldots$ & Rec. No. $31 \quad$ F 204 \\
\hline 2 & $\begin{array}{l}\text { Within the range } 37- \\
41 \mathrm{Mc} / \mathrm{s}: \text { (a) } 38.0 \\
\pm 0.25 \text { (b) } 40.68 \\
\pm 0.25\end{array}$ & 1,2 , and $3 \ldots$ & Rec. No. 32 F 234 \\
\hline 3 & $73.0-74.6 \ldots$ & $2 \ldots$ & F 253 \\
\hline 4 & $79.75-80.25 \ldots$ & $\begin{array}{l}1 \text { and } 3 \text { except } \\
\text { Korea, India, and } \\
\text { Japan. }\end{array}$ & F 261 \\
\hline 5 & $150-153 \ldots$ & 1 & F 286 \\
\hline 6 & $\begin{array}{l}\text { 322-329 (Deuterium } \\
\text { line). }\end{array}$ & No allocation & F 310 \\
\hline 7 & $406-410$ & 1,2 , and $3 \ldots$ & F 317 \\
\hline 8 & $606-614 \ldots$ & 1 and $3 \ldots \ldots$ & $\begin{array}{l}\text { F 332, (Rec. No. 2, } \\
\text { Stockholm, 1961) }\end{array}$ \\
\hline 9 & 1400-1427(Hydrogen line) & 1,2 , and $3 \ldots$ & F 350 \\
\hline 10 & 1645-1675 (OH line) $\ldots$ & No allocation ... & F 354 \\
\hline 11 & $\begin{array}{l}1660-1690 \\
3165-3195 \\
4800-4810 \\
5800-5815 \\
8630-8700\end{array}$ & No allocation & F 354 \\
\hline 12 & $2690-2700 \ldots$ & 1,2 , and $3 \ldots$ & F 365 \\
\hline 13 & $4990-5000 \ldots$ & 1,2 , and $3 \ldots \ldots$ & F 365 \\
\hline 14 & $\begin{array}{c}G c / s \\
10.68-10.70 \\
15.35-15.40 \\
19.3-19.4 \\
31.3-31.5\end{array}$ & $\begin{array}{l}1,2 \text {, and } 3 \\
1,2 \text {, and } 3 \\
1,2 \text {, and } 3 \\
1,2 \text {, and } 3\end{array}$ & $\begin{array}{l}\text { F } 405 \\
\text { F } 405 \\
\text { F } 405 \\
\text { F } 405\end{array}$ \\
\hline
\end{tabular}


6. Appendix B. International Council of Scientific Unions, Inter-Union Commit ee for Frequency Allocations for Radio Âstronomy and Space Science (URSIIAU-COSPAR)

\subsection{Recommendation 1}

The Inter-Union Committee for Frequency Allocations for Radio Astronomy and Space Science considering that

(a) an Extraordinary Administrative Radio Conference is likely to be summoned for 1963 to consider frequency allocations for space communications and research,

(b) all space communication systems will employ orbiting bodies and in some cases belts of orbiting dipoles,

(c) space research devices will employ orbiting bodies,

(d) all these orbiting bodies and dipoles will reflect to any one point on the earth the radio waves from transmitters over a wide area,

(e) waves reflected in this way would interfere with radio astronomical observations, which have to be made with antennas pointing to the sky and with extremely sensitive receiving apparatus,

(f) the possibility that space communication and space research systems would interfere with radio astronomical observations was not envisaged when the agenda for the forthcoming conference was considered in Geneva in 1959, and bearing in mind that

(g) both Radio Astronomy and Space Science, though aimed at different scientific objectives and not being able to share the same radio frequency bands, use very similar techniques,

(h) these same techniques have now made it possible to consider the uses of communication satellite relay systems,

(i) it would seem illogical to discuss these new uses without providing at the same time for the possible continuation of the purely scientific developments which have been at the root of these developments,

(j) both of these new fields of science are actively pursued in various parts of the world, and

(k) at the time of the Geneva Conference in 1959 the full realization of the scope, magnitude and interrelation of these two new activities was not possible, recommends that

Radio Astronomy shall be included in the agenda of the forthcoming Extraordinary Administrative Radio Conference.

\subsection{Recommendation 2}

The Inter-Union Committee for Frequency Allocations for Radio Astronomy and Space Science considering that

(a) according to recommendation No. 32 of the 1959 Conference administrations are asked, when preparing for the next Ordinary Administrative Radio Conference, to consider further the question of frequency allocations for the radio astronomy service,

(b) some frequencies necessary for Radio Astronomy are already allocated to other services on a primary basis recommends that

where no new frequency allocations can be made at the Extraordinary Administrative Radio Conference, actions should be directed at the conference towards a gradual improvement of the position for Radio Astronomy leading up to the reconsideration of adequate frequency allocations at the next Ordinary Administrative Radio Conference.

\section{References}

Adgie, R. L. and J. S. Hey (1957), Intensity of the radio line of galactic deuterium, Nature 179, 370-1.

Blum, E. J., J. F. Denisse, and J. L. Steinberg (1958), Radio astronomy at the Meudon Observatory, Proc. IRE 46 , 39-43.

Bolton, J. G. and G. J. Stanley (1948), Variable source of radio frequency radiation in the constellation of Cygnus, Nature 161, 312-3.

Christiansen, W. N. and J. V. Hindman (1952), A preliminary survey of $1420 \mathrm{Mc} / \mathrm{s}$ line emission from galactic hydrogen, Australian J. Sci. Research 5, 437-455.

Ewen, H. I. and E. M. Purcell (1951), Radiation from galactic hydrogen at $1420 \mathrm{Mc} / \mathrm{s}$, Nature 168, 356.

Findlay, J. W. (1958), Noise levels at the National Radio Astronomy Observatory, Proc. IRE 46, 35-38.

Gemantzev, G. G., K. S. Stankevitch, and V. S. Troitsky (1955). Monokhromaticheskoe radioizluchenie deiteriia na volne 91, $6 \mathrm{sm}$ iz tsentra galaktiki, Dok. Akad. Nauk. USSR 103, 783-786.

Hey, J. S. (1946), Solar radiations in the 4-6 metre radio wavelength band, Nature 15\%, 47-48.

Hey, J. S., S. J. Parsons, and J. W. Phillips (1946), Fluctuations in cosmic radiation at radio frequencies, Nature $\mathbf{1 5 8}$, 234

Hey, J. S. and G. S. Stewart (1946), Derivation of meteor stream radiants by radio reflection methods. Nature 158, 481-2.

Jansky, K. G. (1932), Electrical disturbances of extraterrestrial origin, Proc. Inst. Rad. Eng. 21, 1387-1398.

Maxwell, A., G. Swarup, and A. R. Thompson (1958), The radio spectrum of solar activity, Proc. IRE 46, 142-8.

Mayer, C. H., T. P. McCullough, and R. N. Sloanaker (1958), Measurements of planetary radiation at Centimeter wavelengths, Proc. IRE 46, 260-6.

Mills, B. Y., O. B. Slee, and E. R. Hill (1961), A Catalogue of radio sources between declinations $-50^{\circ}$ and $-80^{\circ}$, Australian J. Phys. 14, 497-507.

Muller, C. A. and J. H. Oort (1951), The interstellar hydrogen line at $1420 \mathrm{Mc} / \mathrm{s}$, and an estimate of galactic rotation, Nature 168, 357-8.

Pawsey, J. L. (1951), Observation of a line in the galactic radio spectrum-radiation from hydrogen at $1420 \mathrm{Mc} / \mathrm{s}$, Nature 168, 358.

Reber, G. (1942), Cosmic Static, Proc. IRE 30, 367-378.

Reber, G. (1944), Cosmic Static, Astrophys. J. 100, 279.

Roman, N. G. and B. S. Yaplee (1958), Radio sources and the Milky Way at 440 Mc, Proc. IRE 46, 199-204.

Ryle, M. (1958), The nature of the cosmic radio sources, Proc. Roy. Soc. 248, 289-308.

Shklowsky, I. S. (1949), Monokhromaticheskoe radioizluchenie galaktiki i vozmozhnost ego nabliudeniia, Astronomicheskii zhurnal 26, 10-14, (Jan-Feb). 
Shklowsky, I. S. (1952), Radiospektroskopiia Galaktiki, Astronomicheskii zhurnal 29, 144-153 (Mar-Apr).

Smith-Rose, R. L. (1961), The allocation of radio frequencies for scientific research, ICSU Review 3, 61-66.

Southworth, G. C. (1945), Microwave radiation from the sun J. Franklin Inst. 239, 285-297.

Stanley, G. J. and R. Price (1956), An investigation of monochromatic radio emission of deuterium from the galaxy, Nature $\mathbf{g} \mathbf{g}, 1221-22$.

van de Hulst, H. C. (1945), Radio waves from space, Ned. Tijdschr. Natuurk. 11, 201 and 210.

Weinreb, S. (1962), A new upper limit to the galactic deuterium-to-hydrogen ratio, Nature 195, 367-368.
Wells, H. W. (1958), Flux measurements of Cassiopeia A and Cygnus A between 18.5 Mc and $107 \mathrm{Mc}$, Proc. IRE 46, 205-208.

International Telecommunication Convention, Geneva, (1959). Radio Regulations, Geneva, Published by the General Secretariat of the International Telecommunication Union, Geneva.

Final Acts of the European VHF/UHF Broadcasting Conference, Stockholm, Published by the ITU, Geneva, 1961.

(Paper 67D2-247) 